Referencia para citar este artículo: Izquierdo-Sánchez, L. \& Ferrer-Ribot, M. (2018). Las experiencias del padre en las Ucin: una revisión desde la perspectiva de género. Revista Latinoamericana de Ciencias Sociales, Niñez y Juventud, 16(1), 55-69. doi:10.11600/1692715x.16102

\title{
Las experiencias del padre en las Ucin: una revisión desde la perspectiva de género*
}

\author{
LAURA IZQUIERDO-SANCHEZ ${ }^{* *}$ \\ Universidad de las Islas Baleares, España. \\ MARÍA FERRER-RIBOT *** \\ Universidad de las Islas Baleares, España.
}

\section{Artículo recibido en febrero 14 de 2016; artículo aceptado en marzo 3 de 2017 (Eds.)}

Resumen (analítico): La mayoría de estudios sobre las implicaciones emocionales y estrategias de afrontamiento de los progenitores ante el nacimiento de un hijo o hija prematuros se centran en la madre. El presente artículo constituye una revisión sistemática a la literatura existente, desde el paradigma cualitativo, acerca de las emociones, sentimientos y estrategias de afrontamiento del padre, y se identifican aspectos relacionados con roles de género en las vivencias y discursos de este. Se constatan diversos perfiles de afrontamiento que generan tres modelos diferenciados de participación en la crianza del neonato prematuro: el equitativo, el desigual y el de rechazo. Se considera que los resultados pueden favorecer la implementación de acciones dirigidas a una mayor inclusión y reconocimiento del padre en los programas de Cuidados Centrados en el Desarrollo de las Unidades de Cuidados Intensivos Neonatales.

Palabras clave: padre, nacimiento prematuro, emociones, estrategias de afrontamiento, análisis de género (Tesauro de MeSH y DeCS).

\section{The experiences of the father in the Nicu: a review from a gender perspective}

- Abstract (analytical): most studies on the emotional implications and confrontations between parents following the birth of premature child focus on the mother. This article details a systematic review of existing literature, from a qualitative paradigm, that cover a father's emotions, feelings and coping strategies. At the same it identifies points related to gender roles in their experiences and discourse. Three coping profiles have been identified, generating three differentiated participation models in the care of a premature child: equal, unequal and rejection. The results of this study could favour the implementation of actions aimed at greater inclusion and recognition of fathers in development-based care in Neo-Natal Intensive Care Units.

Key words: father, premature birth, emotions, coping strategies, gender analysis (MeSH and DeCS Thesaurus).

\footnotetext{
* Este artículo de revisión es una síntesis de la revisión crítica de la literatura llevada a cabo en el marco del "Máster Universitario de Atención a la Primera Infancia: Perspectivas y Líneas de Intervención” de la Universidad de las Islas Baleares, 2015 (Declaración de María A. Riera, directora del Máster, el 12 de septiembre de 2017). Realizado entre agosto de 2014 y septiembre de 2015. Área: sociología; subárea: temas especiales. Este artículo que hace parte de investigación concluida, se enmarca en las líneas de trabajo que el Grupo de Investigación Primera Infancia de la Universidad de las Islas Baleares lleva desarrollando desde hace años en relación al apoyo a la familias en la primera infancia y en este sentido el trabajo abre un tema a partir del cual se podrá seguir profundizando.

** Filóloga, magíster en Primera Infancia por la Universidad de las Islas Baleares, España. Orcid: 0000-0001-8546-7758. Correo electrónico: lisdmt@gmail.com.

*** Psicóloga, doctora en Ciencias de la Educación. Profesora del Departamento de Pedagogía Aplicada y Psicología de la Educación de la Universidad de las Islas Baleares. Orcid: 0000-002-0448-8870. Índice H5: 4. Correo electrónico: maria.ferrer-ribot@uib.es.
} 


\section{As experiências dos pais nas Unidades de Terapia Intensiva Neonatal: uma revisão desde a perspetiva de gênero}

- Resumo (analítico): a maioria dos estudos sobre as implicações emocionais e as estratégias de enfrentamento dos progenitores perante o nascimento de um filho prematuro centram-se na mãe. $O$ presente artigo constitui uma revisão sistemática da literatura existente, desde o paradigma qualitativo, acerca das emoções, sentimentos y estratégias de enfrentamento do pai e, ao mesmo tempo, identificam-se aspectos relacionados com papéis de gênero nas suas vivências e discursos. Constatam-se diversos perfis de enfrentamento que geram três modelos diferenciados de participação na criação do neonato prematuro: o equitativo, o desigual e o de rejeição. Consideramos que os resultados podem favorecer a implementação de ações dirigidas a uma mais vasta inclusão e reconhecimento do pai nos programas de cuidados centrados no desenvolvimento (CCD) e na família das Unidades de Cuidados Intensivos Neonatais.

Palavras chave: pai, nascimento prematuro, emoções, estratégias de enfrentamento, análise de gênero (Tesauro de MeSH e DeCS).

-1. Introducción. -2. Marco teórico. -3. Metodología. -4. Resultados. -5. Discusión. -6. Conclusiones. -7. Lista de referencias.

\section{Introducción}

Las experiencias de bebés prematuros y sus familias en el entorno de una Unidad de Cuidados Intensivos Neonatales (Ucin) son difíciles, pues suponen un alto riesgo de padecer trastornos a nivel de salud mental y de desarrollo (Mesa \& Gómez, 2010). Facilitar el establecimiento de un vínculo afectivo sano y estable es de vital importancia para el desarrollo óptimo de la salud mental en la infancia (Bowlby, 1951, 1999), la adquisición del lenguaje y un sentido de sí mismo (Grossmann \& Grossmann, 2007). Emociones relacionadas con la depresión y el estrés de la madre, además de la culpa, la rabia, la frustración y el temor a la muerte se encuentran suficientemente documentados en la literatura existente (Mesa \& Gómez, 2010). En cambio, la investigación relacionada con las experiencias del padre en este tipo de situaciones ha sido poco o nada abordada (Iriarte \& Carrión, 2013). Tan solo Hollywood y Hollywood (2011), en su revisión bibliográfica, citan los estudios de Hynan (2005), Pohlman (2005), Lindberg, Axelsson y Öhrling (2007), Lundqvist, Westas y Hallström (2007) y Arockiasamy, Holsti y Albersheim (2008) concernientes a experiencias concretas del padre, la mayoría de ellos incluidos en esta revisión.

La Convención sobre los Derechos del Niño (CDN, 1989) reconoce la importancia de

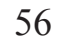

que todos los niños y niñas tengan alimentación, vestido y vivienda, que la atención sanitaria sea universal, que sean protegidos y convivan en un entorno seguro (Oficina del Alto Comisionado para los Derechos Humanos, 2014). Al tratar con prematuros, la presencia parental es todavía más importante, pues se trata de bebés que han sido separados de sus padres al nacer y sometidos a intervenciones que, si bien son indicadas para mantenerlos con vida durante el tiempo necesario hasta su alta hospitalaria, no dejan de ser terapias agresivas. En la actualidad, cada vez más se está permitiendo a los progenitores estar presentes en la Ucin practicando el método madre canguro (MMC). El informe del European Science Foundation Network del 2012 refleja, sin embargo, que tan solo un 40,9\% de los hospitales en España han instaurado el método. En cambio, países como Gran Bretaña, Suecia, Dinamarca o Países Bajos registran cifras del 100\% (Ruz, 2013). Feldman, Rosenthal y Eidelman (2014) y Tessier et al. (2003) comprobaron la eficacia del MMC a largo plazo resaltando que la prontitud en el contacto de la madre y el padre con su hijo o hija así como la permanencia ininterrumpida de uno $o$ ambos progenitores durante el tiempo de hospitalización es determinante para el establecimiento de un buen vínculo y contribuye a la estabilidad de las constantes vitales del bebé.

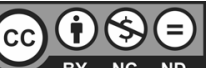

Rev.latinoam.cienc.soc.niñez juv 16 (1): 55-69, 2018 http://revistalatinoamericanaumanizales.cinde.org.co doi: $10.11600 / 1692715 \times .16102$ 
Asimismo, el informe de la Organización Mundial de la Salud (OMS, 2012) Born too soon: The global action report on preterm birth señala que unos 15 millones de bebés en el mundo nacen de manera prematura y hace referencia a la necesidad de inversión y planificación de los recursos humanos para su cuidado tales como la mejora de la capacitación del equipo de salud. Cada año mueren más de un millón de bebés prematuros a causa de complicaciones en el parto. La OMS defiende que la mayoría de esas muertes podrían evitarse con intervenciones activas, eficaces y poco costosas sin necesidad de recurrir a servicios de cuidados intensivos (OMS, 2014). En los países más pobres o en vías de desarrollo las tasas de mortalidad y de secuelas posteriores son más altas que en otros países en los que los avances en medicina permiten implementar intervenciones más eficaces. El Euro-Peristat, cuyo objetivo es monitorear y evaluar la salud materna e infantil en el período perinatal en Europa, cifra en 500 000 el número de nacimientos prematuros al año (Gissler et al., 2010). Entre un 1,5 y un $1,8 \%$ de los bebés nacidos en España fueron bebés prematuros de menos de 32 semanas de gestación, lo cual supone una cifra de 31000 bebés nacidos antes de tiempo (Asociación de Padres de Niños Prematuros, 2015).

En España, los avances técnicos y la investigación científica han permitido desarrollar estrategias de intervención temprana que han supuesto un considerable aumento de la supervivencia del prematuro sin importantes secuelas posteriores de desarrollo. Pero, al igual que cualquier otro niño o niña nacidos a término, en el desarrollo saludable e integral de un bebé prematuro los cuidados de la familia son fundamentales.

Los objetivos de esta revisión son descubrir la literatura existente en la que se describen los sentimientos y estrategias de afrontamiento del padre ante el nacimiento de un hijo o hija prematuros y se exploran los roles de género en la vivencia de su paternidad. Abordar este trabajo desde la perspectiva de género permitirá comenzar a vislumbrar cómo intervienen las distintas formas de sentirse hombre en relación al establecimiento del vínculo paterno-filial en

Rev.latinoam.cienc.soc.niñez juv 16 (1): 55-69, 2018 http://revistalatinoamericanaumanizales.cinde.org.co doi:10.11600/1692715x.16102 un contexto hospitalario. El género masculino no comenzó a ser estudiado hasta la década de los 90, momento en que la psicología, antropología y sociología se dieron cuenta de su invisibilidad tanto para su análisis como para los propios hombres, pues al considerarse lo masculino el "modelo de lo humano" se hacía difícil contemplarlo y problematizarlo para dejar de verlo como el paradigma de lo que debe ser: el modelo androcéntrico de las pautas de conducta (Subirats, 2013). En este sentido, la presente revisión sistemática a la literatura sobre las experiencias paternas desde una perspectiva de género se hace necesaria por no existir hasta el momento trabajo conocido al respecto.

\section{Marco teórico: el modelo feminista}

Históricamente, gran parte de la investigación sobre la paternidad se centra en el enfoque del rol del padre en el desarrollo del niño y de la niña y del impacto de su participación desde una perspectiva psicológica. En los últimos treinta años han aumentado los análisis de las representaciones de género culturales de la paternidad y los procesos interpersonales asociados a cómo construyen y negocian los padres sus identidades parentales (Deeney, Lohan, Spence, \& Parkes, 2012). Asimismo, los estereotipos de género se han ido superando: Ahora sabemos que al padre le cabe un lugar muy especial en la evolución psicológica de sus hijos desde el mismo momento en que estos vienen al mundo; no es un mero comparsa de los supuestos protagonistas (madre-hijo) ni su papel tiene nada de secundario (...). Sabemos, por ejemplo, que el padre es potencialmente capaz de la misma sensibilidad ante el niño que la madre; que el niño establece apego también con él; que la madre tiene una importancia decisiva, pero no exclusiva (Parke, 1981, p. 13).

Por otra parte, a pesar de que en décadas pasadas hubo algunas posturas feministas que postularon que la maternidad es una cuestión inherentemente asociada al biologismo de la mujer, negando el desempeño y deseo del padre (Ehrensaft \& Mansour, 1992), hoy existe un amplio consenso social acerca de la necesaria paternidad responsable (Borisenko, 2007). 
En la actualidad, estamos asistiendo a un proceso de deconstrucción y deslegitimación del pater familias nacido en el contexto del patriarcado (Jiménez, 2004) fundado desde el Antiguo Testamento en el que un Yavéh se erigió como representante de la dominación, autoridad suprema sobre todas las cosas, portador de la vida y de la muerte, guía, protector y norma. Las imágenes y mitos, construcción de las culturas y sociedades occidentales y orientales, fueron introduciendo en el imaginario colectivo esta representación del patriarcado cuyos atributos se concretarían a lo largo de los siglos en el desarrollo de la identidad masculina. El hombre se desarrolló como el sexo fuerte, sin posibilidad de poder mostrar debilidad ni expresar sus sentimientos abiertamente (Pescador, 2004) y, en oposición, la mujer como el sexo débil, dueña de los afectos y las emociones. Esta dicotomía, instaurada a base de prácticas sociales, manifestaciones y constructos culturales, desplegada tanto en la esfera privada como pública, es la que nuestra sociedad posmoderna está comenzando a cuestionar y desautorizar a pesar de que haga tan solo poco más de un siglo que el psicoanálisis freudiano y sus discípulos como Bolwby (1999) y Lacan (1984) contribuyeran a la ratificación y difusión de la imagen cultural de la paternidad como fuente de prohibición y autoridad, y la maternidad como proveedora de afecto (Jiménez, 2004).

Los nuevos padres están emergiendo de las sombras a las que habían sido relegados a causa de la "sacralización del vínculo madre-hijo" presente en las representaciones mentales de los individuos (Jiménez, 2004). Efectivamente, los discursos de las ciencias sociales, antropológicas y psicológicas afirman que el patriarcado es el responsable de tal ensombrecimiento naturalizando su figura con los atributos inherentes de dominación sobre las mujeres y otros grupos sociales y dotando el vínculo materno-filial de cualidades esencialistas, mágicas y naturales (por tanto, inmutables), apartando al padre de las funciones nutricias (emocionales) en la relación con sus hijos e hijas.

El deseo masculino de ejercer roles de cuidado y contención emocional en la crianza está comenzando a emerger en los nuevos discursos sobre la paternidad. Barclay y Lupton (1999) en su estudio longitudinal concluyen que a pesar de que sobresalen nuevas representaciones expresadas por enunciados como "estar presente", "paternidad involucrada" o "vínculo", entre los padres, estos todavía conviven con otros más tradicionales del tipo "ser el proveedor" o "guía" de la familia. De este modo, desean diferenciarse del modelo de padre tradicional heredado, pero organizar su vida ante las nuevas necesidades individuales $\mathrm{y}$ familiares en un contexto de demanda y estructura social todavía patriarcal no está exento de dificultades.

Bonino (2003) escribe sobre las paternidades multiformes y está de acuerdo con la teoría feminista actual de que tanto la maternidad como la paternidad son construcciones sociales y el lugar asignado al padre respecto a sus funciones, deseos y responsabilidades dependerá del contexto y la clase social o la edad.

Paterna, Martínez y Rodes (2005) en su estudio de la revisión feminista sobre la teoría familiar coinciden con Goldner (1989) y HareMustin (1989) que consideran que ambos roles son igualmente necesarios y de igual calidad para el desarrollo del niño o la niña. Por otra parte, los trabajos de Hearn (2004) distinguen entre tipos de investigaciones sobre el hombre y sus masculinidades. En este trabajo nos interesan los Critical Studies of Men (CSM) surgidos de la teoría Queer sobre la investigación de la naturaleza del género del hombre y la masculinidad en las sociedades contemporáneas. Los CSM aportan tres principios fundamentales:

- el género como construcción social

- la masculinidad hegemónica

- desafiar las relaciones de poder entre los géneros.

Ver el género como una construcción social es el logro más importante del feminismo del pasado siglo porque pone en tela de juicio la equivalencia "sexo=género" y en su lugar reconoce que las diferentes culturas y períodos históricos construyen y resignifican el concepto de género atendiendo a las interacciones sociales y a las relaciones de poder entre géneros. Los CSM reconocen que estas relaciones de poder "tienen demasiado género" (Deeney et al., 
2012), es decir, que en las relaciones sociales en las que están implícitas las relaciones de poder existe siempre una mirada masculina, androcéntrica.

Por ello, Hearn (2004) aboga por deconstruir las construcciones hegemónicas de la masculinidad representadas por el abuso de poder sobre mujeres y nos recuerda la necesidad de la transformación de las relaciones de género. A este respecto, Deeney et al. (2012) ve en los CSM el marco teórico idóneo para analizar las experiencias de la paternidad en las Ucin desde una perspectiva crítica de género, pues estas experiencias informan de la naturaleza de las relaciones de género (estereotipos, masculinidad hegemónica...) en las funciones de cuidado.

\section{Metodología}

\section{Diseño metodológico}

El estudio que se presenta puede clasificarse dentro del campo propio de las revisiones sistemáticas de la literatura que según Jesson, Matheson y Lacey (2011) definen una forma de abordar el análisis de la literatura; una forma sistemática y transparente de recopilar, sintetizar y evaluar los resultados de estudios sobre un determinado tema. Dicho análisis se centró en las evidencias existentes, devenidas de estudios cualitativos, acerca de las vivencias del padre de neonatos prematuros en las Ucin.

Se realizó una búsqueda bibliográfica en PubMed, Scopus, Science Direct, Dialnet y otras fuentes como la Revista Infancia $y$ Aprendizaje: Journal for the Study of Education and Development, la Enciclopedia sobre el desarrollo de la primera infancia y la editorial americana Sage Publications utilizando los descriptores "padre", "nacimiento prematuro", "paternidad"; y "father", "Intensive Care Unit", "fathers perceptions", "fathers experiences", "premature newborn", "nicu". Se emplearon, asimismo, variaciones de los términos con operadores booleanos.

Se incluyeron aquellas referencias de estudios más actualizados llevados a cabo en los últimos diez años previos a la elaboración de esta revisión (2005-2015), y se seleccionaron investigaciones de paradigma cualitativo con muestras por conveniencia escritos en castellano, inglés o portugués. La selección de estudios de corte cualitativo se justifica por ser esta metodologia más cercana a la descripción de vivencias. Las revisiones - a excepción de la de Iriarte y Carrión (2013) por tratarse de una fuente primaria cercana a la presente revisiónfueron excluidas para evitar la contaminación de los sesgos derivados de la subjetividad de otros investigadores, así como las tesis y libros por ser inabarcable su contenido en este trabajo. De los resultados obtenidos $(n=51)$ se llevó a cabo una evaluación de calidad con el objetivo de controlar todos los aspectos relevantes en cuanto a su validez. Para ello se adaptó una plantilla elaborada por Cano, González y Cabello (2010) que evalúa la calidad de los estudios cualitativos a partir del instrumento desarrollado por el Critical Appraisal Skills Programme (Casp) del Institute of Health Sciences de la Universidad de Oxford.

De los 51 estudios iniciales se seleccionaron finalmente 15. Los motivos para ser eliminados en base a los criterios de evaluación de calidad fueron no asegurar estrategias de credibilidad, no reflexionar sobre la transferibilidad a otros contextos, no plantear claramente los objetivos o no quedar explícita la pregunta de investigación.

\section{Análisis del contenido}

Tras la lectura crítica de los estudios se elaboró una tabla con información sobre los objetivos, la muestra, el tipo de diseño, y los resultados (Tabla 1). El análisis del contenido se llevó a cabo con el desarrollo de un marco temático, teniendo como referencia los objetivos y el marco conceptual de esta investigación, y se indexaron los datos en las dimensiones descritas en el apartado de resultados. Para organizar, estructurar y codificar toda la información se utilizaron mapas mentales y conceptuales (Soria, Giménez, Fanlo, \& Escanero, 2007). En la Figura 1 se presenta la síntesis de los resultados de la búsqueda bibliográfica. 
Figura 1. Síntesis de los Resultados de la Búsqueda Bibliográfica.

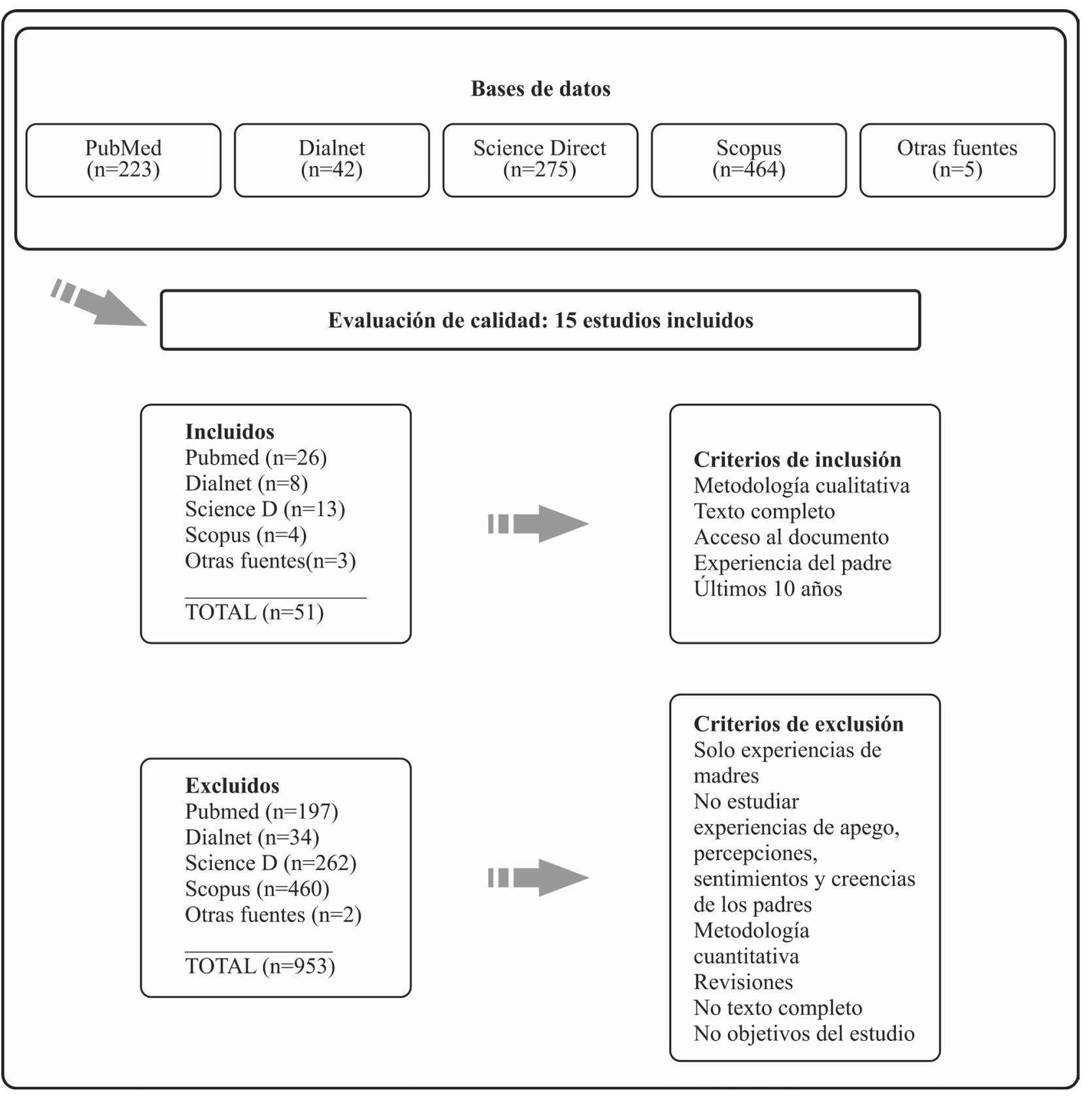


Tabla 1. Sintesis de los estudios analizados.

\begin{tabular}{|c|c|c|c|c|}
\hline Artículo & Objetivo & Muestra & Tipo de diseño & Resultados \\
\hline $\begin{array}{l}\text { Arockiasamy } \\
\text { et al. (2008) }\end{array}$ & $\begin{array}{c}\text { Entender los } \\
\text { mecanismos de } \\
\text { apoyo y experiencia } \\
\text { del padre } \\
\end{array}$ & $\begin{array}{c}16 \\
\text { padres }\end{array}$ & Descriptivo & $\begin{array}{l}\text { Sensación de falta de control. Trabajar y recibir } \\
\text { ayuda les ayudó a recuperarlo y llevar a cabo } \\
\text { roles de protectores, padres, compañeros y } \\
\text { proveedores }\end{array}$ \\
\hline $\begin{array}{l}\text { Blomqvist et } \\
\text { al. (2012) }\end{array}$ & $\begin{array}{c}\text { Describir las } \\
\text { experiencias } \\
\text { paternas con MMC }\end{array}$ & 7 padres & Descriptivo & $\begin{array}{l}\text { El MMC les proporcionó seguridad, } \\
\text { empoderamiento e implicación. Pasar tiempo } \\
\text { con sus bebés les dio sensación de control }\end{array}$ \\
\hline $\begin{array}{l}\text { Deeney et al. } \\
\text { (2012) }\end{array}$ & $\begin{array}{l}\text { Explorar las } \\
\text { relaciones de } \\
\text { género de poder } \\
\text { derivadas de la } \\
\text { masculinidad } \\
\text { hegemónica }\end{array}$ & $\begin{array}{c}21 \\
\text { padres }\end{array}$ & $\begin{array}{l}\text { Diseño de } \\
\text { investigación } \\
\text { longitudinal }\end{array}$ & $\begin{array}{l}\text { Reconocen la "fortaleza" como una } \\
\text { característica estereotipada masculina heredada } \\
\text { de la cual se resienten, pero a la vez les sirve } \\
\text { de soporte. Paradoja entre ser un buen padre y } \\
\text { a la vez tener que seguir proveyendo el hogar. } \\
\text { Algunos hombres o no quisieron o fueron } \\
\text { incapaces de cambiar las relaciones de poder } \\
\text { que sitúan a la mujer como cuidadora principal }\end{array}$ \\
\hline $\begin{array}{l}\text { Feeley et al. } \\
\text { (2013) }\end{array}$ & $\begin{array}{l}\text { Describir los } \\
\text { patrones de } \\
\text { participación } \\
\text { durante la } \\
\text { hospitalización } \\
\end{array}$ & $\begin{array}{c}18 \\
\text { padres }\end{array}$ & $\begin{array}{l}\text { Estudio de caso } \\
\text { múltiple }\end{array}$ & $\begin{array}{l}\text { Tres tipos de patrones: implicación afectiva } \\
\text { simétrica a la madre, sacralización del } \\
\text { vínculo madre-hijo y actividades indirectas de } \\
\text { participación; y reacios a participar }\end{array}$ \\
\hline $\begin{array}{l}\text { Helth y Jarden } \\
\text { (2013) }\end{array}$ & $\begin{array}{c}\text { Explorar las } \\
\text { experiencias de los } \\
\text { padres con el MMC }\end{array}$ & 5 padres & $\begin{array}{l}\text { Estudio } \\
\text { exploratorio } \\
\text { con enfoque } \\
\text { fenomenológico- } \\
\text { hermenéutico } \\
\end{array}$ & $\begin{array}{l}\text { El MMC les proporciona confianza, placer y } \\
\text { competencia en los cuidados }\end{array}$ \\
\hline $\begin{array}{l}\text { Hollywood y } \\
\text { Hollywood } \\
\quad(2011)\end{array}$ & $\begin{array}{l}\text { Explorar las } \\
\text { experiencias de los } \\
\text { padres }\end{array}$ & 5 padres & $\begin{array}{l}\text { Estudio } \\
\text { exploratorio } \\
\text { con enfoque } \\
\text { fenomenológico- } \\
\text { hermenéutico }\end{array}$ & $\begin{array}{l}\text { Sentimientos de impotencia y ansiedad durante } \\
\text { la hospitalización. Ventajas y desventajas de } \\
\text { obtener información. Atención centrada en } \\
\text { la familia y participación. No sentían que su } \\
\text { papel estaba valorado. Pocas redes de apoyo } \\
\text { disponibles. Restricciones en el trabajo }\end{array}$ \\
\hline $\begin{array}{l}\text { Hugill et al. } \\
\quad(2013)\end{array}$ & $\begin{array}{c}\text { Explorar las } \\
\text { experiencias } \\
\text { emocionales de los } \\
\text { padres }\end{array}$ & $\begin{array}{c}10 \\
\text { padres }\end{array}$ & $\begin{array}{l}\text { Estudio cualitativo } \\
\text { longitudinal } \\
\text { con enfoque } \\
\text { etnográfico }\end{array}$ & $\begin{array}{l}\text { Existe una "emoción silenciosa" en los } \\
\text { padres menos expresivos. Algunos intentaron } \\
\text { protegerse ocultando sus emociones, } \\
\text { especialmente de los trabajadores sanitarios, } \\
\text { buscando desahogarse en privado. Otros } \\
\text { controlaban sus emociones para proteger a sus } \\
\text { compañeras y cumplir con su auto-asignado } \\
\text { papel de protector }\end{array}$ \\
\hline $\begin{array}{l}\text { Lindberg et al. } \\
\qquad(2007)\end{array}$ & $\begin{array}{l}\text { Describir las } \\
\text { experiencias de los } \\
\text { padres }\end{array}$ & 8 padres & Descriptivo & $\begin{array}{l}\text { Les confiere una sensación de control necesaria } \\
\text { para su estabilidad emocional el hecho de } \\
\text { sentirse protectores tanto de la madre como } \\
\text { del niño, pero siguen necesitando empatía y } \\
\text { comprensión ya que sienten iguales miedos y } \\
\text { momentos de estrés }\end{array}$ \\
\hline
\end{tabular}




\begin{tabular}{|c|c|c|c|c|}
\hline $\begin{array}{l}\text { Lindberg et al. } \\
\qquad(2008)\end{array}$ & $\begin{array}{l}\text { Describir las } \\
\text { experiencias de los } \\
\text { padres }\end{array}$ & 8 padres & Descriptivo & $\begin{array}{l}\text { Los padres necesitan tomarse el tiempo } \\
\text { necesario para llegar a sentirse competentes, } \\
\text { seguros y confiados, y para ello juega un papel } \\
\text { importante el contacto continuo y prolongado } \\
\text { con el bebé. Hay un cambio en sus valores y } \\
\text { prioridades en la vida }\end{array}$ \\
\hline $\begin{array}{l}\text { Lindberg y } \\
\text { Engström } \\
\text { (2013) }\end{array}$ & $\begin{array}{l}\text { Describir las } \\
\text { experiencias de los } \\
\text { padres }\end{array}$ & 8 padres & Descriptivo & $\begin{array}{l}\text { Los padres luchan para que el equipo de salud } \\
\text { los reconozca como parte de la familia }\end{array}$ \\
\hline $\begin{array}{l}\text { Lundqvist et } \\
\text { al. (2007) }\end{array}$ & $\begin{array}{l}\text { Describir las } \\
\text { experiencias de los } \\
\text { padres }\end{array}$ & $\begin{array}{c}13 \\
\text { padres }\end{array}$ & $\begin{array}{l}\text { Diseño cualitativo } \\
\text { con enfoque } \\
\text { hermenéutico- } \\
\text { fenomenológico }\end{array}$ & $\begin{array}{l}\text { La experiencia de los padres parte de sensaciones } \\
\text { de distancia emocional inmediatamente después } \\
\text { del parto hacia progresivas sensaciones de } \\
\text { mayor proximidad }\end{array}$ \\
\hline $\begin{array}{l}\text { Lundqvist et } \\
\text { al. (2014) }\end{array}$ & $\begin{array}{l}\text { Describir las } \\
\text { experiencias de los } \\
\text { padres al cabo de } \\
\text { los tres años }\end{array}$ & 8 padres & $\begin{array}{l}\text { Estudio } \\
\text { longitudinal } \\
\text { con enfoque } \\
\text { hermenéutico- } \\
\text { fenomenológico }\end{array}$ & $\begin{array}{l}\text { Proceso de reorganización vital: lucha diaria, } \\
\text { empoderamiento y crear una base segura }\end{array}$ \\
\hline $\begin{array}{l}\text { Santos et al. } \\
\qquad(2012)\end{array}$ & $\begin{array}{l}\text { Comprender las } \\
\text { experiencias de los } \\
\text { padres }\end{array}$ & 9 padres & $\begin{array}{l}\text { Descriptivo- } \\
\text { exploratorio }\end{array}$ & $\begin{array}{l}\text { Sentimientos de preocupación, ansiedad, } \\
\text { angustia, inseguridad, estrés. Conflicto ante el } \\
\text { mandato social de ser proveedor y el deseo de } \\
\text { pasar tiempo con el bebé }\end{array}$ \\
\hline $\begin{array}{l}\text { Sweet y } \\
\text { Darbyshire } \\
(2009)\end{array}$ & $\begin{array}{l}\text { Explorar las } \\
\text { experiencias } \\
\text { paternas con la } \\
\text { lactancia }\end{array}$ & 7 padres & $\begin{array}{l}\text { Estudio } \\
\text { longitudinal } \\
\text { con enfoque } \\
\text { fenomenológico- } \\
\text { interpretativo }\end{array}$ & $\begin{array}{l}\text { Todos apoyaron la lactancia de su compañera, } \\
\text { pero en los casos de biberón mostraron un papel } \\
\text { activo. Les proporcionó placer e intimidad }\end{array}$ \\
\hline $\begin{array}{l}\text { Pohlman } \\
(2005)\end{array}$ & $\begin{array}{c}\text { Explorar el } \\
\text { significado del } \\
\text { trabajo para los } \\
\text { padres y el impacto } \\
\text { de este en su } \\
\text { transición hacia la } \\
\text { paternidad }\end{array}$ & 9 padres & $\begin{array}{l}\text { Estudio } \\
\text { longitudinal } \\
\text { con enfoque } \\
\text { fenomenológico- } \\
\text { interpretativo }\end{array}$ & $\begin{array}{l}\text { Los padres tendían a volver al trabajo poco } \\
\text { después del nacimiento y con un sentimiento } \\
\text { aumentado de celos. Tienen necesidad de } \\
\text { trabajar por no existir baja remunerada. } \\
\text { Los hombres ocultan sus emociones a sus } \\
\text { compañeras, pero saben que la represión } \\
\text { emocional dificulta la gestión del estrés }\end{array}$ \\
\hline
\end{tabular}

\section{Resultados}

Los 15 estudios analizados fueron llevados a cabo en Canadá $(n=2)$, Suecia $(n=6)$, Norte de Irlanda $(\mathrm{n}=2)$, Dinamarca $(\mathrm{n}=1)$, Reino Unido $(n=1)$, Australia $(n=1)$, EE. UU. $(n=1)$ y Brasil $(n=1)$. Los resultados se relacionan con las dimensiones que a continuación se describen.

\section{Estrategias de afrontamiento}

Las investigaciones analizadas describen que, para la mayoría de los padres, lograr la estabilidad emocional ante la crisis vivida sintiéndose protectores de la madre y del bebé, y obtener tanto la empatía como la comprensión por parte de los demás - familia y equipo de salud-, les sirvió como estrategia de afrontamiento para recuperar el sentimiento de falta o pérdida de control (Arockiasamy et al., 2008; Lindberg et al., 2007). "La primera vez que lo vi me vine abajo, no sabía qué hacer, no había hecho esto antes" (Hugill, Letherby, Reid, \& Lavender, 2013, p. 657).

Otro factor señalado en los estudios como importante de recuperación del control 
de la situación y seguridad en sí mismos fue poder mantenerse informados acerca de los acontecimientos - tanto técnicos como del progreso del bebé-, así como su deseo de salvaguardar el contacto con el neonato (Blomqvist, Rubertsson, Kylberg, Jöreskog, \& Nyqvist, 2012; Santos, Silva, Santana, \& Santos, 2012). "Informar al padre sobre lo que está pasando (...) les hace sentir que son alguien" (Arockiasamy et al., 2008, p. 218).

Además de las estrategias de autogestión emocional y los apoyos externos para afrontar la situación, el trabajo fue un tema recurrente en sus vidas. Para unos se trató de una vía de escape y descanso del momento estresante: "Al mismo tiempo, algunos de los padres consideran el tiempo fuera del hospital como una ventaja, 'un respiro', donde podían recargar" (Helth \& Jarden, 2013, p.119). Y otros lo vivieron como una forma de presión social y paradoja al no tener elección entre ir a trabajar o quedarse con sus bebés en la unidad (Helth \& Jarden, 2013; Hollywood \& Hollywood, 2011). Esta falta real de libertad se manifestó en el hecho de que renunciar a este podía suponerles tanto la pérdida como la disminución de las posibilidades de crecimiento profesional (Hollywood \& Hollywood, 2011).

Durante la hospitalización de su hijo en la Ucin, el padre vivencia una situación de estrés intenso pues teme perder su empleo, ya sea por faltar al trabajo, retrasarse o verse obligado a pedir permisos, y ve en la continuación de sus actividades laborales la seguridad financiera que necesita proporcionar a la familia (Santos et al., 2012, p. 791).

En cambio, para otros padres el aspecto laboral de sus vidas supuso una gran oportunidad para estar más en contacto con el bebé y su pareja. Este fue el caso de aquellos que disfrutaron de una baja remunerada por paternidad (Lindberg, Axelsson, \& Öhrling, 2008; Lundqvist, Hellström-Westas, \& Hallström, 2014) o que pudieron elegir cuándo trabajar o no: "Por el momento, puedo elegir las horas que quiero trabajar, pero sé que mi situación no es la norma aquí" (Deeney et al., 2012, p. 4).

Finalmente, algunos padres identificaron de pleno su rol paternal con su rol de proveedores, es decir, para ellos ser padres significaba ser el responsable del sustento económico familiar (Pohlman, 2005) bien por convicciones y creencias propias, bien porque seguían el modelo heredado de sus propios padres.

\section{Emociones y sentimientos}

En relación a las emociones, esta revisión muestra que el apoyo externo familiar y del equipo de salud de la Ucin, la posibilidad de tener contacto continuado con el bebé ya fuera por el hecho de dar el biberón o de practicar el contacto piel con piel, la información completa y coherente sobre el estado de salud del prematuro y la actitud positiva y comprometida del padre suscitaron en ellos emociones de confianza, competencia, placer y seguridad en sí mismos, y empoderamiento. Es decir, se valoraron como buenos y verdaderos padres (Blomqvist et al., 2012; Helth et al., 2013; Lindberg et al., 2007; Lundqvist et al., 2007).

En cambio, los estudios señalan que sentimientos de impotencia, ansiedad, miedos, frustración (Hollywood \& Hollywood, 2011; Lindberg \& Engström, 2013; Lundqvist et al., 2007; Santos et al., 2012), angustia y contradicciones (Hugill et al., 2013; Lundqvist et al., 2007), insatisfacción (Blomqvist et al., 2012) y culpabilidad por sentirse incapaces de afrontar y hacerse cargo de la nueva situación familiar (Lundqvist et al., 2014) también les embargaron. Las causas de esta emocionalidad fueron básicamente dos: la falta de información y apoyo, y la falta de reconocimiento en entornos excesivamente feminizados.

\section{Roles de género}

Los estudios muestran que algunos padres aceptan sin cuestionar el rol heredado de la cultura patriarcal que dicta que ellos son los principales proveedores de la seguridad $\mathrm{y}$ economía familiares. La función protectora y proveedora, sin embargo, los mantiene alejados del contacto con su bebé (Arockiasamy et al., 2008; Feeley, Sherrard, Waitzer, \& Boisvert, 2013; Hugill et al., 2013; Pohlman, 2005), bien porque no tienen tiempo, bien porque 
creen que los cuidados del hijo deben recaer exclusivamente en la madre.

Sin embargo, otros padres luchan en su fuero interno por superar la paradoja entre ser buenos padres y compañeros y, a la vez, proveedores, como señalan Deeney et al. (2012), Lundqvist et al. (2014) y Santos et al. (2012). La función hegemónica de saberse protectores les resulta inquietante, pues desean pasar más tiempo con sus hijos y participar de los cuidados. Son conscientes de que están contribuyendo a perpetuar los roles heredados, pero estos también son una vía de afrontamiento para defenderse de la emoción dolorosa de la separación (Deeney et al., 2012; Hugill et al., 2013). Directamente relacionado con esto último se encuentra la fortaleza, que ellos reconocen como una cualidad heredada estereotipada, pero que a la vez les sirve de mucho apoyo: "Quiero decir, si puedo manejar esto, puedo manejar mucho más" (Lundqvist et al., 2007, p. 128).
La sociedad les obliga a mantenerse fuertes y ser el sostén emocional tanto de la pareja como del hijo, aunque alejados de ellos (Deeney et al., 2012; Hugill et al., 2013). Los padres desean un cambio en las estructuras hegemónicas heredadas socialmente y consideran un desafío que la situación que viven les haya obligado a trabajar para lograr el equilibrio entre su rol parental y los mandatos culturales de lo que significa ser hombre (Deeney et al., 2012; Helth, \& Jarden, 2013). Así, algunos padres, a pesar de que ven el reto y se sienten incómodos ante la disyuntiva, opinan que no tienen elección y acaban asumiendo el mandato sin cuestionarlo.

En los estudios consultados se refleja también la convivencia de formas de participación en la crianza del neonato prematuro que se han agrupado en los modelos que se presentan en la Figura 2:

Figura 2. Modelos de Participación en la Crianza.

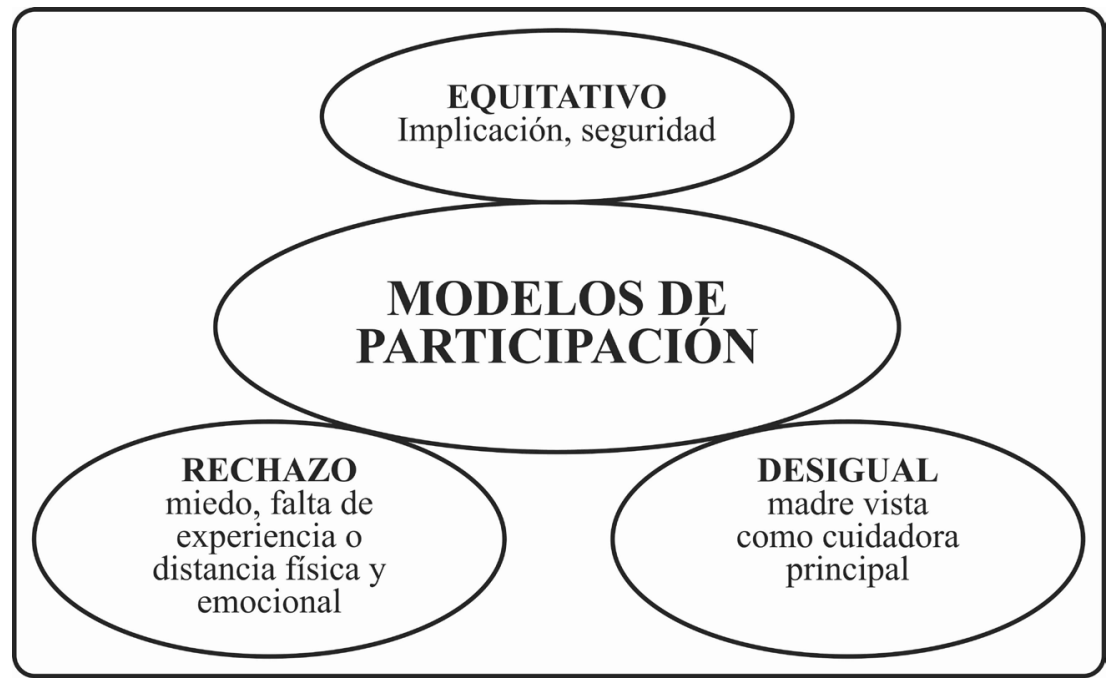

- Modelo equitativo en cuanto a cuidados e implicación afectiva, lo cual aumenta las sensaciones de empoderamiento, satisfacción y seguridad. "Compartimos todo por igual. Nunca hubo ninguna discusión acerca de eso; cuando uno de nosotros estaba cansado, el otro se hizo cargo" (Blomqvist et al., 2012, p. 4). También se señala que: "Con los bebés yo no me limito.
Soy capaz de hacerlo todo. Y si no soy capaz voy a aprender o alguien me va a enseñar, por lo que no hay diferencia entre mi participación y la de la madre" (Feeley et al., 2013, p.75). Estos padres exigen a los equipos de salud una atención centrada en la familia que favorezca su participación (Hollywood \& Hollywood, 2011). 
- Modelo desigual: la madre es vista como la cuidadora principal (Deeney et al., 2012; Feeley et al., 2013) por parte de los padres. En algunos casos es el equipo de salud quien lo ve así (Hollywood \& Hollywood, 2011; Lindberg \& Engström, 2013). En este sentido, los padres ven diferencia de trato, sintiéndose inferiores y poco competentes en un entorno muy feminizado (Lundqvist et al., 2007).

- Modelo de rechazo: ya sea por el miedo, la falta de experiencia o la distancia física y emocional, algunos padres rechazan explícitamente participar directamente en los cuidados: "Tenía miedo de hacerle daño. Era tan pequeña, tan frágil. Tengo miedo de hacerle daño, de que se me caiga. Simplemente, no quiero hacerlo (cogerla en brazos)" (Feeley et al., 2013, p. 77).

\section{Discusión}

En los resultados de esta revisión se observa que el apego que puede formar el padre con su bebé prematuro está condicionado por factores de diversa índole, a saber: emocionales, sociales, culturales, personales y de género. La separación repentina, a pesar de suponer un desajuste emocional para ellos y de trasladarlos a emociones angustiosas y temerosas, no necesariamente tiene por qué derivar en un trastorno en el establecimiento del vínculo.

Hemos visto que en muchos casos los padres construyen sus identidades parentales en base a la tradición hegemónica heredada $\mathrm{y}$, efectivamente, el patriarcado influye en los sentimientos encontrados y paradójicos de aquellos que luchan por deshacerse de los mandatos culturales y sociales de género de ser proveedores y protectores para poder abandonarse a la emocionalidad, a la vulnerabilidad de saberse dolidos por la situación y al deseo de establecer contacto íntimo con su hijo o hija. En este sentido, surgen en las investigaciones analizadas aspectos emocionales como confianza, placer o autoestima cuando estos padres pueden acceder a sus bebés amorosamente y llevar a cabo funciones de cuidado y contención emocional, roles que tradicionalmente están condicionados por el formato de género femenino (Levinton, 2000), además de angustia, insatisfacción y culpabilidad.

En su revisión, Núñez, Berrocal, Rodríguez y Postigo (2008) tratan de explorar la verdad o el mito que existe detrás de las pautas de sociabilización emocional según el género. Entre sus hallazgos mencionan los estudios que utilizaron pruebas de autoinforme (que analizan la propiocepción emocional en hombres y mujeres) y en algunos casos encuentran diferencias que se pueden relacionar estrechamente con emociones de género heredadas: las mujeres se perciben como más diestras a la hora de comprender y manejar sus emociones y los hombres lo hacen "en relación con el control de impulsos y la tolerancia al estrés" (Núñez et al., 2008, p. 463). Esto último se puede aplicar a la necesidad de recuperar el control como estrategia para lograr la estabilidad emocional. Ciertamente, ni todas las mujeres ni todos los hombres están moldeados por los mandatos de género heredados, y también estos últimos están biológicamente preparados para desarrollar sentimientos de compasión y ternura. Tal y como se apuntaba en el apartado del marco teórico en lo concerniente a los tres focos de investigación principales de los CSM se ha podido ver que algunos hombres de los estudios analizados están comenzando a deconstruir los mandatos sociales de género, además de cuestionar las relaciones de poder entre estos al proponer modelos de participación en el cuidado de sus hijos e hijas más equitativos.

En la actualidad, la mayoría de las investigaciones sobre experiencias maternas y paternas en las Ucin de Europa y América tienen como objetivo específico generar la suficiente información entre los equipos de salud de los hospitales para que se implementen protocolos de actuación centrados en el cuidado familiar, y no tan solo en la madre y el bebé. Así, hemos visto en los resultados que alguno de los entornos excesivamente feminizados hacía que los padres se sintieran fuera de lugar e incompetentes.

A partir de las investigaciones llevadas a cabo se observa la suficiente evidencia que apunta claramente hacia un camino a seguir: 
contrariamente a los estereotipos culturales heredados del patriarcado, el padre entra en contacto con su parte más emocional y vulnerable frente a situaciones dramáticas, como es el nacimiento de un hijo o hija de manera prematura. Las estrategias para afrontar el dolor y la situación varían entre las que podrían situarse en la esfera de los roles tradicionales masculinos: recurrir al trabajo, sentirse empoderados controlando la información, ser protectores y proveedores, y los roles tradicionalmente vinculados a lo femenino: ser cuidadores de sus hijos e hijas, necesidad de ser incluidos en el sistema familiar, dejarse sentir emociones de angustia, soledad y preocupación. Pero esta división es antinatural puesto que impide ver las naturalezas masculina y femenina como similares.

Cabe hacer mención a las limitaciones de los estudios analizados en cuanto al tipo de población de la muestra en las investigaciones: hombres en su mayoría caucásicos, europeos o americanos, de clase social media. Este es un sesgo importante a tener en cuenta pues los resultados hubieran variado entre población de distinta cultura y clase social.

\section{Conclusiones}

Tras el análisis de los estudios revisados es posible concluir que las experiencias de estos padres ante el nacimiento de un hijo o hija prematuros describen sentimientos vinculados con la competencia, valorización y empoderamiento en entornos que favorecen el contacto de este con el neonato; en cambio, la falta de apoyo social y laboral (permisos de paternidad inexistentes o insuficientes) -en este sentido la investigación actual sugiere cambios en el terreno de lo político y lo social que favorecen el ejercicio más igualitario de la paternidad (Suárez, 2015), si bien estos avances son todavía lentos-, contribuyen a generar sentimientos de minusvalía personal y dificultades en las estrategias de afrontamiento ante la separación. Asimismo, los aspectos identificados en los estudios con los roles de género dan a conocer la convivencia de diferentes modelos de crianza que van desde el ejercicio del rol de padre más tradicional y menos implicado en el vínculo afectivo hasta el más involucrado y afectuoso con su bebé.

Por último, la revisión realizada en este trabajo muestra la evidencia acerca de la importancia del apoyo psicosocial de todo el sistema familiar (Miguel, Briones, \& Prats, 2012). En este sentido, los resultados y conclusiones de esta investigación pueden favorecer la implementación de programas de Cuidados Centrados en el Desarrollo (CCD) en las Ucin en los que se incluya con mayor relevancia al padre en la toma de contacto y crianza con su hijo o hija prematuros.

\section{Lista de referencias}

Arockiasamy, V., Holsti, L., \& Albersheim, S. (2008). Fathers' experiences in the neonatal intensive care unit: A search for control. Pediatrics, 121(2), 215-222. doi:10.1542/ peds.2007-1005

Asociación de Padres de Niños Prematuros (2015). El 75\% de los ingresos hospitalarios de neonatos son de bebés prematuros. Recuperado de: http://www.aprem-e.org/ el-75-de-los-ingresos-hospitalarios-de-neonatos-son-de-bebes-prematuros/\#more-1348.

Barclay, L., \& Lupton, D. (1999). The experiences of new fatherhood: a sociocultural analysis. Journal of Advanced Nursing, 29(4), 1013-1020. doi:10.1046/ j.1365-2648.1999.00978.x

Blomqvist, Y. T., Rubertsson, C., Kylberg, E., Jöreskog, K., \& Nyqvist, K. H. (2012). Kangaroo Mother Care helps fathers of preterm infants gain confidence in the paternal role. Journal of Advanced Nursing, 68(9), 1988-1996. doi:10.1111/ j.1365-2648.2011.05886.x

Bonino, L. (2003). Las nuevas paternidades. Cuadernos de Trabajo Social, 16, 171-182.

Borisenko, J. (2007). Fatherhood as a personality development factor in men. The Spanish Journal of Psychology, 10(1), 82-90.

Bowlby, J. (1951). Maternal care and mental health. Bulletin of the World Health Organization, (3), 355-534.

Bowlby, J. (1999). Vínculos afectivos: 
formación, desarrolloy pérdida. Barcelona: Gedisa.

Cano, A., González, T., \& Cabello, J. B. (2010). Plantilla para ayudarte a entender un estudio cualitativo. En CASPe (Ed.), Guías CASPe de lectura crítica de la literatura médica (Cuaderno III. pp. 3-8). Alicante: CASPe.

Deeney, K., Lohan, M., Spence, D., \& Parkes, J. (2012). Experiences of fathering a baby admitted to neonatal intensive care: A critical gender analysis. Social Science \& Medicine, 75 (6), 1106-111. doi:10.1016/j.socscimed.2012.04.018

Ehrensaft, D. \& Mansour, M. (1992). Las feministas pelean contra (por) padres. Debate Feminista, 6, pp. 93-118.

Feeley, N., Sherrard, K., Waitzer, E., \& Boisvert, L. (2013). The father at the bedside: Patterns of involvement in the Nicu. The Journal of Perinatal \& Neonatal Nursing, 27(1), 72 80. doi:10.1097/JPN.0b013e31827fb415

Feldman, R., Rosenthal, Z., \& Eidelman, A. I. (2014). Maternal-preterm skin-toskin contact enhances child physiologic organization and cognitive control across the first 10 years of life. Biological Psychiatry, 75(1), 56-64. doi:10.1016/j. biopsych.2013.08.012

Gissler, M., Mohangoo, A. D., Blondel, B., Chalmers, J., Macfarlane, A., Gaizauskiene, A., \& Zeitlin, J. (2010). Perinatal health monitoring in Europe: Results from the Euro-Peristat Project. Informatics for Health and Social Care, 35(2), 64-79. doi: $10.3109 / 17538157.2010 .492923$

Goldner, V. (1989). Generation and gender: Normative and covert hierarchies. En M. McGoldrick, C. M. Anderson, \& F. Walsh (Eds.), Women and families (pp. 42-60). New York: Norton.

Grossmann, K., \& Grossmann, K. E. (2007). Impacto del apego a la madre y al padre durante los primeros años, en el desarrollo psicosocial de los niños hasta la adultez temprana. Montreal: Centre of Excellence for Early Childhood Development.

Hare-Mustin, R. (1989). The problem of gender in family therapy theory. En M. McGoldrick, C. M. Anderson, \& F. Walsh
(Eds.), Women and families (pp. 61-77). New York: Norton.

Hearn, J. (2004). From hegemonic masculinity to the hegemony of men. Feminist Theory, 5(1), 49-72. doi:10.1177/1464700104040813.

Helth, T., \& Jarden, M. (2013). Fathers' experiences with the skin-to-skin method in Nicu: Competent parenthood and redefined gender roles. Journal of Neonatal Nursing, 19(3), 114-121. doi:http://dx.doi. org/10.1016/j.jnn.2012.06.001

Hollywood, M., \& Hollywood, E. (2011). The lived experiences of fathers of a premature baby on a neonatal intensive care unit. Journal of Neonatal Nursing, 17(1), 32-40. doi:http://dx.doi.org/10.1016/j. jnn.2010.07.015

Hugill, K., Letherby, G., Reid, T., \& Lavender, T. (2013). Experiences of fathers shortly after the birth of their preterm infants. Journal of Obstetric, Gynecologic, \& Neonatal Nursing, 42(6), 655-663. doi:http://dx.doi. org/10.1111/1552-6909.12256

Hynan, M. (2005). Supporting fathers during stressful times in the nursery: An evidencebased review. Newborn and Infant Nursing Reviews, 5(2), 87-92. doi: http://dx.doi. org/10.1053/j.nainr.2005.03.006

Iriarte, A., \& Carrión, M. (2013). Experiencias de los padres de grandes prematuros en la unidad de cuidado intensivo neonatal: revisión sistemática de la evidencia cualitativa. Metas de Enfermería, 16(2), 20-25.

Jesson, J. K., Matheson, L., \& Lacey, F. M. (2011). Doing your literature review: Tradictional and systematic techniques. Londres: Sage.

Jiménez, A. B. (2004). La paternidad en entredicho. Gaceta de Antropología, 20(19), 1-19. doi:http://hdl.handle. net/10481/7270

Lacan, J. (1984). Les complexes familiaux. París: Navarin.

Levinton, N. (2000). El superyó femenino: la moral en las mujeres. Madrid: Biblioteca Nueva.

Lindberg, B., Axelsson, K., \& Öhrling, K. (2007). The birth of premature infants: Experiences from the father's perspective. 
Journal of Neonatal Nursing, 13(4), 142149. doi:10.1016/j.jnn.2007.05.004

Lindberg, B., Axelsson, K., \& Öhrling, K. (2008). Adjusting to being a father to an infant born prematurely: experiences from Swedish fathers. Scandinavian Journal of Caring Sciences, 22(1), 79-85. doi:10.1111/ j.1471-6712.2007.00563.x

Lindberg, I., \& Engström, Å. (2013). A qualitative study of new fathers' experiences of care in relation to complicated childbirth. Sexual \& Reproductive Healthcare, 4(4), 147-152. doi:http://dx.doi.org/10.1016/j. srhc.2013.10.002

Lundqvist, P., Hellström-Westas, L., \& Hallström, I. (2014). Reorganizing life: A qualitative study of fathers' lived experience in the 3 years subsequent to the very preterm birth of their child. Journal of Pediatric Nursing, 29(2), 124-131. doi:http://dx.doi.org/10.1016/j. pedn.2013.10.008

Lundqvist, P., Westas, L. H., \& Hallström, I. (2007). From distance toward proximity: fathers lived experience of caring for their preterm infants. Journal of pediatric nursing, 22(6), 490-497. doi:10.1016/j. pedn.2007.04.008

Mesa, A. M., \& Gómez, A. C. (2010). La mentalización como estrategia para promover la salud mental en bebés prematuros. Revista Latinoamericana de Ciencias Sociales, Niñez y Juventud, 8(2), 835-848. Recuperado de: http://www. redalyc.org/pdf/773/77315155005.pdf.

Miguel, M. J. C., Briones, A. B. E., \& Prats, S. G. (2012). Enfermería neonatal: cuidados centrados en la familia. Enfermería Integral: Revista Cientifica del Colegio Oficial de ATS de Valencia, 98, 36-40.

Núñez, M. T. S., Berrocal, P. F., Rodríguez, J. M., \& Postigo, J. M. L. (2008). ¿Es la inteligencia emocional una cuestión de género?: socialización de las competencias emocionales en hombres y mujeres y sus implicaciones. Electronic Journal of Research in Educational Psychology, 6(15), 455-474.

Oficina del Alto Comisionado para los Derechos Humanos (2014). Convención sobre los
Derechos del Niño. Recuperado de: http:// www2.ohchr.org/spanish/law/crc.htm.

Organización Mundial de la Salud. (2004). Método madre canguro: guía práctica. Recuperado de: http://www.who.int/ reproductivehealth/publications/maternal_ perinatal_health/9241590351/

Organización Mundial de la Salud. (2012). Centro de prensa. Nacimientos prematuros. Datos y cifras. Recuperado de: http://www. who.int/mediacentre/factsheets/fs363/es/

Organización Mundial de la Salud. (2014). Temas de salud: salud del niño. Recuperado de: http://www.who.int/topics/child health/es/

Parke, R. D. (1981). El papel del padre. Madrid: Morata.

Paterna, C., Martínez, C., \& Rodes, J. (2005). Creencias de los hombres sobre lo que significa ser padre. R. interam. Psicol, 39, 2.

Pescador, E. (2004). Masculinidades y adolescencia. En C. Lomas (Comp.), Los chicos también lloran: identidades masculinas, igualdad entre los sexos y coeducación, (pp. 113-146). Barcelona: Paidós Educador.

Pohlman, S. (2005). The primacy of work and fathering preterm infants: Findings from an interpretive phenomenological study. Advances in Neonatal Care, 5(4), 204-216. doi:10.1016/j.adnc.2005.03.002

Ruz, R. (2013, 8 de mayo). El método madre canguro podría ahorrar a la sanidad española 200 millones. Madrid: $A B C$. Recuperado de: http://www.abc.es/ sociedad/20130508/abci-mama-canguroahorro-salud-201305071726.html.

Santos, L. M., Silva, C. L. D. S., Santana, R. C. B. D., \& Santos, V. E. P. (2012). Vivências paternas durante a hospitalização do recém-nascido prematuro na Unidade de Terapia Intensiva Neonatal. Rev. bras. enferm, 65 (5), 788-794. doi:http://dx.doi. org/10.1590/S0034-71672012000500011

Soria, M., Giménez, I., Fanlo, A. J., \& Escanero, J. F. (2007). El mapa conceptual: una nueva herramienta de trabajo. Diseño de una práctica para fisiología. Investigación 
Educativa. Zaragoza: Universidad de Zaragoza.

Suárez, M. M. (2015). Maternidad, paternidad y Estado: los derechos de conciliación como desarrollo del derecho a la igualdad entre mujeres y hombres. Ius et Veritas, 19(39), 224-243.

Subirats, M. (2013). Forjar un hombre, moldear una mujer. Barcelona: Aresta.

Sweet, L., \& Darbyshire, P. (2009). Fathers and breastfeeding very-low-birthweight preterm babies. Midwifery, 25(5), 540553. doi:http://dx.doi.org/10.1016/j. midw.2007.09.001

Tessier, R., Cristo, M. B., Vélez, S., Giron, M., Nadeau, L., de Calume, Z. F. ... \& Charpak, N. (2003). Kangaroo Mother Care: A method for protecting high-risk low-birthweight and premature infants against developmental delay. Infant Behavior and Development, 26(3), 384-397. doi:10.1016/ S0163-6383(03)00037-7 